\title{
Median nail dystrophy
}

\author{
Morteza Khodaee MD MPH, Naomi Kelley BS, Sabrina Newman MD
}

Cite as: CMAJ 2020 December 14;192:E1810. doi: 10.1503/cmaj.201002

A 34-year-old man presented to his primary care physician with a 20-year history of painless bilateral thumbnail lesions. The patient had no history of nail trauma or infection. Both thumbs had a central linear depression in a fir tree pattern, surrounded by parallel transverse ridges (Figure 1). There was no surrounding erythema or warmth, and the nails were not tender to touch. His other fingernails and toenails were unaffected. We diagnosed median nail dystrophy.

Median nail dystrophy, also known as dystrophyia unguis, mediana canaliformis, median canaliform dystrophy or solenonychia, is an uncommon nail condition affecting men and women equally. Believed to be related to microtrauma involving the nail matrix, median nail dystrophy is often symmetric, and most commonly affects the thumbs. ${ }^{1-3}$ Common differential diagnoses for longitudinal nail defects include habit tic deformity, subungual skin tumours, digital mucous cyst and trachyonychia (rough nails). ${ }^{4-6} A$ thorough history should focus on occupational injuries and habits. Other conditions to consider include onychomycosis, lichen planus, Darier disease and psoriasis. ${ }^{3-5}$ To rule out onychomycosis, testing using a potassium hydroxide or optical fluorescence preparation, or a fungal culture are suggested. Signs of a fungal infection include fungal white or yellow chromonychia, onychauxis (thickened nails) and onycholysis with subungual debris., ${ }^{4,6}$ Lichen planus is very unlikely if there are no other skin or mucosal lesions (e.g., Wickham striae).,5 Lichen planus-related nail splits tend to be irregular and are not typically associated with ridging or macrolunulae., ${ }^{3,5}$

Although median nail dystrophy has been treated with topical corticosteroids, tacrolimus and tazarotene, there is little compelling evidence that the nail changes can be reversed. ${ }^{1-3}$ Our patient elected not to pursue any interventions as the condition was purely cosmetic.

\section{References}

1. Kota R, Pilani A, Nair PA. Median nail dystrophy involving the thumb nail. Indian J Dermatol 2016;61:120.

2. Pathania V. Median canaliform dystrophy of Heller occurring on thumb and great toe nails. Med J Armed Forces India 2016;72:178-9.

3. Richert B, Caucanas M, André J. Diagnosis using nail matrix. Dermatol Clin 2015;33:243-55.

4. Tully AS, Trayes KP, Studdiford JS. Evaluation of nail abnormalities. Am Fam Physician 2012;85:779-87.

5. Biesbroeck LK, Fleckman P. Nail disease for the primary care provider. Med Clin North Am 2015;99:1213-26.

6. Wollina U, Nenoff P, Haroske G, et al. The diagnosis and treatment of nail disorders. Dtsch Arztebl Int 2016;113:509-18.

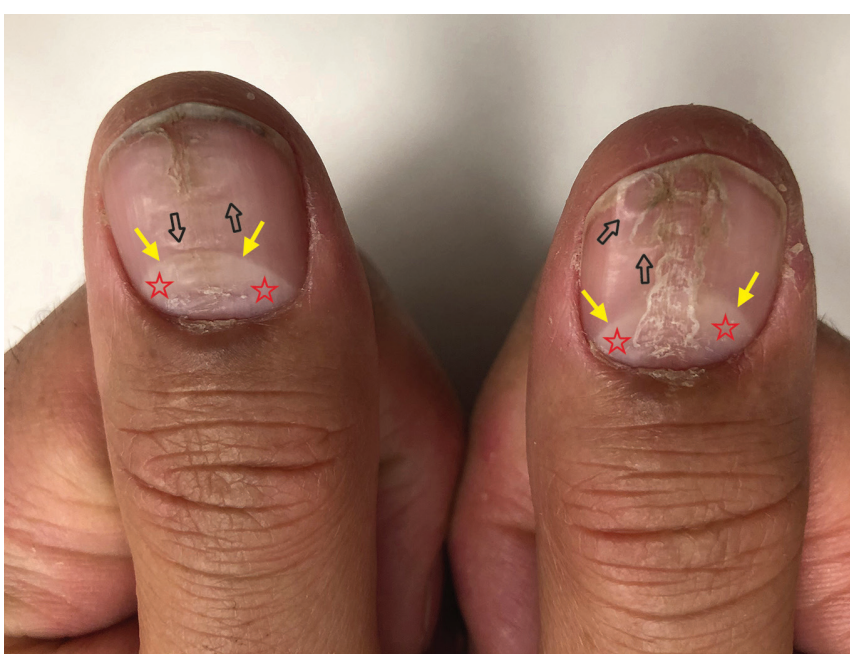

Figure 1: Median nail dystrophy in a 34-year-old man. Thumbs with central feathered longitudinal lamellar split, surrounded by parallel transverse ridges (open arrows) of the nail plates, macrolunulae (shown by the white areas [red stars] between the nail fold and the remainder of the nail plate [yellow arrows]) and associated focal proximal nail fold hyperpigmentation and hyperkeratosis.

\section{Competing interests: None declared.}

This article has been peer reviewed.

The authors have obtained patient consent.

Affiliations: Department of Family Medicine and Orthopedics (Khodaee), University of Colorado School of Medicine, AFW Clinic, Denver, Colo.; University of Colorado School of Medicine (Kelley); Department of Dermatology (Newman), University of Colorado School of Medicine, Aurora, Colo.

Correspondence to: Morteza.khodaee@cuanschutz.edu

Clinical images are chosen because they are particularly intriguing, classic or dramatic. Submissions of clear, appropriately labelled highresolution images must be accompanied by a figure caption. A brief explanation ( 300 words maximum) of the educational importance of the images with minimal references is required. The patient's written consent for publication must be obtained before submission. 